\title{
Qualidade de vida no período puerperal: importância e satisfação
}

\author{
Quality of life in the puerperal period: importance and satisfaction
}

Paulo César Condeles ${ }^{1}$, Sarah Gazarra Ferreira da Silva ${ }^{1}$, Dyego Brito Fernandes ${ }^{1}$, Bibiane Dias Miranda Parreira ${ }^{1}$, Marina Carvalho Paschoini ${ }^{1}$, Mariana Torreglosa Ruiz ${ }^{1}$

Objetivo: mensurar a qualidade de vida de mulheres que estão vivenciando o puerpério. Métodos: estudo transversal, realizado com 103 puérperas, a partir da aplicação do instrumento "Índice de Qualidade de Vida", de Ferrans e Powers. Resultados: a média do índice de qualidade de vida geral foi de 24,77 pontos. Dentre os domínios, o que teve a maior pontuação foi o família e o mais afetado foi o socioeconômico. Presença de dor e condições socioeconômicas foram os principais motivos de insatisfação das puérperas, enquanto que a saúde dos filhos e da família foi sinalizada como o item mais importante e de maior satisfação. Conclusão: as puérperas apresentaram altos escores de qualidade de vida. Contudo, o domínio socioeconômico foi o mais comprometido e o domínio família o que apresentou melhores escores, indicando importância e satisfação diante do nascimento e da configuração familiar.

Descritores: Qualidade de Vida; Período Pós-Parto; Saúde da Mulher; Enfermagem Obstétrica.

Objective: to measure the quality of life of women who are experiencing the puerperium. Methods: crosssectional study performed with 103 postpartum women, based on the application of the Ferrans and Powers Quality of Life Index. Results: the average overall quality of life index was 24.77 points. Among the domains, the highest score was obtained in the family domain, and the most affected was the socioeconomic. Presence of pain and socioeconomic conditions were the main reasons for postpartum dissatisfaction, while the most important and most satisfying item was Health of the child and of the family. Conclusion: the mothers had high quality of life scores. However, the socioeconomic domain was the most compromised and the family domain presented the best scores, indicating the importance and satisfaction linked to birth and family configuration.

Descriptors: Quality of Life; Postpartum Period; Women's Health; Obstetric Nursing.

${ }^{1}$ Universidade Federal do Triângulo Mineiro. Uberaba, MG, Brasil. 


\section{Introdução}

Segundo a Organização Mundial da Saúde, a saúde não é simplesmente a inexistência de doenças, mas é um conceito complexo que compreende um estado de completo bem-estar físico, mental e social afetado por diversos fatores, entre eles, as condições de trabalho, questões de gênero, estilo de vida, exclusão social, violência e violação de direitos humanos ${ }^{(1-2)}$. Essa mesma organização ${ }^{(2)}$ definiu o termo qualidade de vida como a percepção do indivíduo sobre sua posição na vida e na sociedade na qual está inserido, incluindo suas metas, expectativas, padrões e preocupações. Este conceito abrange aspectos físicos e psicológicos da saúde, assim como a independência, crença, relações sociais e fatores ambientais ${ }^{(1)}$. A qualidade de vida contempla o indivíduo como um todo, em seus vários domínios, e mudanças ocorridas na saúde podem influenciá-la diretamente.

O período puerperal ou pós-parto é um período cronológico e individualmente variável e inicia-se com a expulsão da placenta, findando aproximadamente na sexta semana após o parto. Contudo, as repercussões geradas pela gestação no organismo materno podem persistir por até um ano após o nascimento ${ }^{(3)}$.

Neste período, as mulheres sofrem uma série de transformações, de ordem física, psicológica e social, podendo cursar com agravos e doenças ${ }^{(4)}$. Essas transformações também são diretamente afetadas pelo estresse provocado pelo parto, responsabilidade de cuidar de um recém-nascido e as alterações no seu cotidiano $^{(5)}$.

De forma que se pode deduzir que mudanças na saúde das puérperas poderão acarretar consequências para a qualidade de vida destas mulheres. Estudo realizado na Holanda, com mulheres na sexta semanas pós-parto, apontou que os domínios físico e mental foram os mais comprometidos neste período ${ }^{(6)}$. Ainda, estudo conduzido no interior do Estado de São Paulo ressaltou que, no puerpério imediato (até duas horas após o parto), mulheres apresentaram baixos escores de qualidade de vida, com comprometimento dos do- mínios capacidade funcional, aspecto físico e $\operatorname{dor}^{(7)}$.

Diante dos resultados que apontam importantes alterações na qualidade de vida no período puerperal e considerando a melhoria e a ampliação da atenção à saúde da mulher em seus componentes de pré-natal, parto e puerpério preconizadas pela Rede Cegonha $^{(8)}$, faz-se necessário conhecer a mulher em todas as suas dimensões, considerando suas características sociodemográficas, história obstétrica, condições de saúde e hábitos de vida, para que enfermeiros e outros profissionais da saúde possam prestar assistência de qualidade pautada na singularidade dos indivíduos e na humanização $0^{(7-9)}$.

A realização deste estudo justifica-se pela necessidade de se conhecer as dimensões afetadas da qualidade de vida destas mulheres, que vivem um período complexo e de transformações em suas vidas, e que se fomentem ações de promoção, proteção e recuperação da saúde no período puerperal.

Este estudo teve como objetivo mensurar a qualidade de vida de mulheres que estão vivenciando o puerpério.

\section{Métodos}

Trata-se de um estudo transversal, realizado com mulheres no período puerperal. Os dados foram coletados no período de março a dezembro de 2017, em um hospital de ensino, no município de Uberaba, Estado de Minas Gerais, Brasil.

A amostra foi constituída por mulheres que estavam vivenciando o puerpério (na primeira semana ou até 90 dias após o parto), que tiveram retorno de puerpério e/ou do neonato agendado na instituição; que sabiam ler e escrever; que estivessem em condições para responder ao questionário independentemente da idade (puérperas com idade inferior a 18 anos tiveram o Termo de Consentimento Livre e Esclarecido assinado pelos pais e/ou responsáveis legais e assentiram seu consentimento).

Constituíram-se critérios de exclusão: puérperas que tiveram como desfecho da gestação o aborto, 
óbito fetal, natimorto ou óbito neonatal, assim como puérperas contrarreferenciadas para retorno puerperal em Unidade Básica de Saúde ou Estratégia Saúde da Família onde realizaram consultas pré-natais e mulheres que tiveram o parto assistido em outras instituições. Desse modo, atenderam a tais critérios e foram incluídas 103 puérperas, que representaram também a população deste estudo, atendendo aos critérios de inclusão e exclusão.

Os dados foram obtidos por meio de entrevista com as puérperas e/ou extraídos dos prontuários das mesmas. Todas as mulheres foram abordadas na sala de espera momentos antes da consulta puerperal e/ ou neonatal nos ambulatórios do hospital de ensino e, entrevistadas antes ou após a realização da mesma, no período determinado para a coleta dos dados.

As puérperas foram convidadas a responder ao questionário que abordava dados sociodemográficos, condições de saúde, história obstétrica, dados do nascimento e do recém-nascido. Esse questionário foi elaborado pelos próprios pesquisadores, baseado nas informações que constam nos prontuários institucionais e testado mediante estudo-piloto, mostrando-se adequado para responder às variáveis de interesse do estudo.

Para mensurar os escores de Qualidade de Vida, foi utilizada a versão genérica do Índice de Qualidade de Vida, desenvolvido pelas pesquisadoras Carol Estwing Ferrans e Marjorie Powers, da Universidade de Chicago e Illinois - EUA. O instrumento possui duas partes: a primeira associa-se à satisfação, onde cada questão varia em uma escala de pontuação de um (muito insatisfeito) a seis (muito satisfeito), e a segunda reflete a importância atribuída aos seus itens, também em uma escala de pontuação que varia de um (sem nenhuma importância) a seis (muito importante $)^{(10-11)}$. Trata-se de um instrumento validado, traduzido para o português do Brasil e disponível para uso livre e gratuito, assim como a sua sintaxe.

$O$ instrumento mensura a qualidade de vida em geral e subdivide seus itens em quatro domínios para os quais gera as respectivas pontuações do escore. São eles: saúde e funcionamento; psicológico e espiritual; família e socioeconômico. A pontuação varia na faixa de $0-30$, para todas as versões, sendo que, quanto maior o escore final, melhor a qualidade de vida ${ }^{(10-11)}$.

Após a coleta, os dados foram codificados e realizou-se dupla digitação e validação, armazenando-os em planilhas do Excel ${ }^{\circledR}$. Posteriormente, foram transportados para o Statistical Package for the Social Sciences (versão 23) e analisados por estatística descritiva: frequência, medida de tendência central (média) e medidas de dispersão (desvio-padrão, mínimo e máximo).

Todas as participantes foram esclarecidas a respeito do estudo. 0 estudo foi aprovado pelo Comitê de Ética em Pesquisa da Universidade Federal do Triângulo Mineiro sob o parecer no 1.774 .885 .

\section{Resultados}

Ao caracterizar as 103 puérperas entrevistadas, a média de idade foi de $25,81( \pm 6,51)$ anos, variando de 14 a 42 anos. Destas mulheres, 43 (41,7\%) declaravam-se pardas, $43(41,7 \%)$ não exerciam atividade remunerada, 50 (48,5\%) eram católicas, 40 (38,8\%) tinham completado o Ensino Médio e 51 (49,5\%) possuíam renda familiar de um a dois salários mínimos. Em sua maioria, eram casadas 67 (65,0\%), possuíam casa própria $58(57,3 \%)$ e residiam no próprio município 70 (68,0\%).

Quanto às condições de saúde e hábitos, nove $(8,7 \%)$ relataram etilismo, seis $(5,7 \%)$, tabagismo e uma puérpera referiu fazer uso de drogas ilícitas; 31 $(30,1 \%)$ possuíam doenças prévias à gestação, sendo mais frequentes a hipertensão arterial, oito $(25,8 \%)$ casos e o hipotireoidismo, seis (22,6\%). Entretanto, $73(70,9 \%)$ gestações cursaram com alguma doença, sendo as mais frequentes: hipotireoidismo 19 (26,0\%); síndromes hipertensivas 15 (20,5\%); diabetes gestacional oito $(10,9 \%)$; anemia e sífilis (quatro casos, ambas com percentual de 5,5\%) e depressão, 
dois $(2,7 \%)$ casos. Com relação à história obstétrica, os dados são apresentados na Tabela 1.

Tabela 1 - História obstétrica das 103 puérperas entrevistadas

\begin{tabular}{lcccc}
\hline \multirow{2}{*}{ Variáveis } & Média & \multicolumn{4}{c}{$\begin{array}{c}\text { Desvio- } \\
\text { Padráni- }\end{array}$} & Máxi- & mo \\
\hline Número de gestações & 2,46 & 1,54 & 1 & 9 \\
Número de partos & 2,23 & 1,41 & 1 & 9 \\
Número de abortos & 0,24 & 0,47 & 0 & 2 \\
Número de consultas de pré-natal & 8,14 & 2,45 & 0 & 14 \\
Idade gestacional (em semanas) & 38,54 & 1,53 & 33 & 42 \\
Peso ao nascimento (em gramas) & 3090,58 & 636,85 & 1260 & 4495 \\
Apgar 1ํ minuto de vida & 8,40 & 1,31 & 2 & 10 \\
Apgar 5 minuto de vida & 8,86 & 0,68 & 6 & 10 \\
Retorno puerperal (em dias) & 36,10 & 18,69 & 8 & 125 \\
\hline
\end{tabular}

Em relação ao tipo de parto, 49 (47,6\%) foram cesáreos e, desses, 20 (40,8\%) foram indicados por alterações na vitalidade fetal; 11 (2,4\%), por descompensação de patologia materna e 11 (22,4\%), por iteratividade. Já o parto normal com episiotomia correspondeu a $29(28,2 \%)$ e o parto normal sem intervenções a $25(24,3 \%)$ que, somados, totalizaram a maioria das vias de parto 54 (52,5\%). Na amostra, não houve nenhuma puérpera submetida a parto fórceps.

No momento da entrevista, $93(90,3 \%)$ relataram estar amamentando, 73 (70,9\%), em aleitamento exclusivo e $20(19,4 \%)$, em aleitamento misto; 66 $(64,1 \%)$ consideraram o ato de amamentar ótimo; $26(24,2 \%)$ relataram ocorrência de trauma mamilar, sendo mais frequentes a escoriação $16(15,5 \%)$ e a fissura nove $(8,7 \%)$ casos. 0 retorno da puérpera na instituição variou de oito a 90 dias, com média de retorno em $36,1( \pm 18,7)$ dias pós-parto.

A pontuação média obtida nos escores de qualidade de vida total foi de $24,77( \pm 3,21)$ e, dentre os domínios do Índice de Qualidade de Vida, o que teve a maior pontuação foi o família e o mais afetado foi o social e econômico. 0 domínio psicológico/espiritual foi o que apresentou a maior variação (Tabela 2).
Tabela 2 - Índice de qualidade de vida total e por domínios das 103 puérperas entrevistadas

\begin{tabular}{lcccc}
\hline Índice de Qualidade de Vida & Média & \multicolumn{1}{c}{$\begin{array}{c}\text { Desvio- } \\
\text { Padrão }\end{array}$} & Mínimo & Máximo \\
\hline Total & 24,77 & 3,21 & 13,89 & 30 \\
Saúde e funcionamento & 24,51 & 4,00 & 12,79 & 30 \\
Social e econômico & 22,33 & 4,46 & 10,38 & 30 \\
Psicológico/espiritual & 25,80 & 4,25 & 4,64 & 30 \\
Família & 27,55 & 2,40 & 20,4 & 30 \\
\hline
\end{tabular}

Ao analisar os aspectos da qualidade de vida abordados no instrumento, com relação à satisfação das puérperas, as questões que tiveram menor pontuação foram: a satisfação com o lar $(3,27)$; com o apoio recebido $(4,22)$; com as condições sociais $(4,30)$; com os vizinhos $(4,34)$ e com a dor $(4,50)$. Apresentaram maiores escores de satisfação: com seu (s) filho (s) $(5,92)$; com sua fé em Deus $(5,89)$; com a felicidade da sua família $(5,83)$; com a possibilidade de viver como gostaria $(5,82)$ e vislumbrando a possibilidade de um futuro feliz $(5,81)$.

Já em relação à importância atribuída aos aspectos da qualidade de vida, as questões que tiveram menor pontuação foram: é importante não ter preocupações $(4,73)$; a relação com os vizinhos $(4,92)$; o relacionamento com os amigos $(5,25)$; ter um trabalho/emprego $(5,36)$; e ser você mesma $(5,48)$. Foi atribuída maior importância aos aspectos: saúde da família $(5,89)$ e dos filhos $(5,89)$; a felicidade da família $(5,85)$; sua saúde $(5,85)$; não ter dores $(5,82)$ e os cuidados com sua saúde $(5,81)$.

\section{Discussão}

As limitações do estudo foram quanto ao método utilizado, por se tratar de estudo com desenho transversal, e no que tange à validade externa, uma vez que os dados não podem ser generalizados para outras realidades e não podem ser estabelecidas relações causais.

Este estudo contribuiu ao revelar aspectos afetados na qualidade de vida das mulheres que estão 
passando por um período complexo e de transformações, e visa, a partir da compreensão destes fatores, a proporcionar atenção qualificada, humana, respeitando as singularidades do período e de cada mulher, e subsidiar ações de saúde individuais e coletivas voltadas para esse público.

A pontuação média obtida nos escores de qualidade de vida geral foi semelhante a de outro estudo, realizado no Ceará, com mulheres que estavam vivenciando este mesmo período ${ }^{(12)}$.

Semelhantemente, em outros estudos conduzidos no Brasil ${ }^{(10)}$ e em outros países ${ }^{(13-14)}$, que utilizaram instrumentos diferentes para a coleta de dados, observam-se altos escores de qualidade de vida em geral. No entanto, estudos realizados na Holanda ${ }^{(6)}$, Canadá( ${ }^{(15)}$ e na Suécia ${ }^{(16)}$ apontaram maior comprometimento nos escores de qualidade de vida, principalmente nos domínios físico e mental. Porém, destaca-se que ambos os estudos foram realizados com puérperas que apresentaram doenças na gestação e/ou puerpério, o que pode ter influenciado negativamente os escores de qualidade de vida ${ }^{(6,15-16)}$.

Neste estudo, o domínio de qualidade de vida obteve a maior média, e a menor média de escore foi o família, o social e econômico, respectivamente, corroborando outra pesquisa com puérperas realizada no Brasil $^{(10)}$. Fortalecem-se esses resultados em uma pesquisa realizada no Centro-Norte da Nigéria, com 550 puérperas entre a sexta e oitava semanas pós-parto, sobre qualidade de vida e depressão, que mostrou que o domínio relação sociais foi o mais bem pontuado, demonstrando a importância da rede social para essas mulheres. Neste mesmo estudo, o domínio que obteve menor pontuação foi o ambiental ${ }^{(17)}$.

Destaca-se a importância do apoio familiar para a puérpera neste momento da vida. Esta associação fica evidente a partir dos resultados de um estudo realizado na Coreia do Sul, que mostrou correlação negativa entre a satisfação com o apoio do companheiro e sinais de depressão; condições de saúde e sintomas no puerpério(5). Da mesma forma, estudo realizado no sul da Índia, com 274 puérperas entre a sexta e oitava semanas pós-parto, evidenciou asso- ciação entre maiores escores de qualidade de vida e o suporte social da puérpera, incluindo o apoio do marido, familiares, amigos e pais. Com relação ao domínio social e econômico, este mesmo estudo do sul da Índia mostrou que o nível socioeconômico apresentou correlação positiva com os maiores escores ${ }^{(18)}$.

Estudo que avaliou qualidade de vida com puérperas adolescentes apontou resultados semelhantes aos deste estudo: o domínio psicológico/espiritual obteve a maior variação nos escores. Houve maior satisfação com os seguintes aspectos da qualidade de vida: "a sua fé em Deus", "felicidade de sua família" e "filhos". Essa mesma pesquisa mostrou que, dentre as questões que obtiveram menor pontuação, se destaca o fato de não ter um trabalho, tanto na satisfação quanto na importância, contribuindo para os menores escores do domínio social e econômico. Ressalta-se que o desemprego no período pós-parto pode limitar a independência das mulheres ${ }^{(10)}$.

Contrapõe-se um estudo realizado na Coreia do Sul, com 148 mulheres no período pós-parto, que mostrou que as puérperas que retornaram ao trabalho apresentavam piores desempenhos no autocuidado, demonstrando influência da sobrecarga de atividades na saúde das mulheres ${ }^{(5)}$.

Estudo de intervenção realizado com 168 puérperas, em que a intervenção consistiu na realização de cinco consultas de enfermagem, apontou maior taxa de aleitamento materno exclusivo; maior adesão ao exame colpocitológico e uso de sulfato ferroso e melhorias na cobertura vacinal materna ${ }^{(19)}$, indicando a importância do acompanhamento puerperal e seus benefícios na saúde materna.

Ressalta-se a importância do apoio familiar e social no período puerperal, assim como a necessidade de avaliar a mulher em suas singularidades neste período. Compreender aspectos e intervir diante das necessidades podem prevenir agravos e doenças. Assim, cabe ao enfermeiro e demais profissionais da saúde detectar alterações que comprometam a saúde destas mulheres a fim de assegurar melhores condições para elas, seus filhos e sua família. 


\section{Conclusão}

As puérperas apresentaram altos escores de qualidade de vida. Contudo, o domínio socioeconômico foi o mais comprometido neste período e o domínio família foi o que apresentou melhores escores, indicando importância e satisfação diante do nascimento e da configuração familiar. A presença de dor e as condições socioeconômicas foram os principais motivos de insatisfação das puérperas, enquanto que a saúde dos filhos e da família foi sinalizada como o item mais importante e de maior satisfação para as mesmas.

\section{Agradecimentos}

A Fundação de Amparo à Pesquisa do Estado de Minas Gerais e a Universidade Federal do Triângulo Mineiro pelo apoio financeiro, através de Bolsa de Iniciação Científica.

\section{Colaborações}

Condeles PC, Silva SGF e Ruiz MT contribuíram com concepção do projeto, análise e interpretação dos dados, redação do artigo, revisão crítica relevante do conteúdo intelectual e aprovação final da versão a ser publicada. Fernandes DB, Parreira BDM e Paschoini MC contribuíram com a aprovação final da versão a ser publicada.

\section{Referências}

1. Organização Pan-Americana de Saúde. OPAS/ OMS apoia governos no objetivo de fortalecer e promover a saúde mental da população [Internet]. 2016 [citado 2019 Jul 22]. Disponível em: https:// www.paho.org/bra/index.php?option=com_co ntent\&view=article\&id=5263:opas-oms-apoiagovernos-no-objetivo-de-fortalecer-e-promovera-saude-mental-da-populacao\&Itemid $=839$

2. World Health Organization. WHOQOL: Measuring Quality of Life [Internet]. 2019 [cited Jun 24, 2019]. Available from: https://www.who.int/ healthinfo/survey/whoqol-qualityoflife/en/
3. Maciel LP, Costa JCC, Campor GMB, Santos NM, Melo RA, Diniz LFB. Mental disorder in puerpério: risks and mechanisms of counseling for the promotion of health. Rev Pesqui Cuid Fundam Online. 2019; 11(4):1096-102. doi: http://dx.doi. org/10.9789/2175-5361.2019.v11i4.1096-1102

4. Mazzo MHSN, Brito RS. Nursing instrument to attend mothers who recently gave birth in primary care. Rev Bras Enferm. 2016; 69(2):316-25. doi: dx.doi.org/10.1590/0034-7167.2016690215I

5. Lee JY, Hwang JY. A study on postpartum symptoms and their related factors in Korea. Taiwan. J Obstet Gynecol. 2015; 54(4):355-63. doi: http://dx.doi. org/10.1016/j.tjog.2014.04.030

6. Prick BW, Bijlenga D, Jansen AJG, Boers KE, Scherjon AS, Koopmans CM, et al. Determinants of health-related quality of lyfe in the postpartum period after obstetric complication. Eur J Obstet Gynecol Reprod Biol. 2015; 185:88-95. doi: http://dx.doi.org/10.1016/j.ejogrb.2014.11.038

7. Soler DR, Ponce MAZ, Soler ZASG, Wysocki AD. Quality of life in the purperium: assessment in the immediate, late and remote postpartum period. Rev Enferm UFPE online [Internet]. 2015 [cited Jun 02, 2019]; 12(9):1093-101. Available from:https://periodicos.ufpe.br/revistas / revistaenfermagem/article/view/10812/11989

8. Alves AG, Martins CA, Pinho ES, Almeida NAM, Tobias GC. Teaching practice of the nurse in the stork network based of the historical-cultural theory. Rev Enferm UFPE online [Internet]. 2017 [cited Jul 23, 2019]; 11(9):3330-7. Available from:https://periodicos.ufpe.br/revistas / revistaenfermagem/article/view/110230

9. Olegário WKB, Fernandes LTB, Medeiros CMR. Validation of ICNP nursing diagnoses for assistance to women during postpartum. Rev Eletr Enferm. 2015; 17(3):1-7. doi: http://dx.doi.org/10.5216/ ree.v17i3.31502

10. Ferrans CE, Powers M. Quality of life index [Internet]. 2019 [cited Apr 23, 2019]. Available from: http://qli.org.uic.edu/index.htm

11. Ferreira FM, Haas VJ, Pedrosa LAK. Quality of life of adolescents after maternity. Acta Paul Enferm. 2013; 26(3):245-9. doi: http://dx.doi. org/10.1590/S0103-21002013000300007 
12. Oliveira MF, Parker L, Ahn H, Catunda HLO, Bernardo EBR, Oliveira MF, et al. Maternal predictors for quality of life during the postpartum in brazilians mothers. Health. 2015; 7:371-80. doi: http://dx.doi.org/10.4236/health.2015.73042

13. El Sayed SLM, Emarah HAM. Relationship between mode of birth and quality of life for women's health during postpartum period. Am J Nurs. 2019; 8(2):75-80. doi: https://dx.doi.org/10.11648/j. ajns.20190802.16

14. Miller CM, Ramachandran B, Akbar K, Carvalho B, Butwick AJ. The impact of postpartum hemoglobin levels on maternal quality of life after delivery: a prospective exploratory study. Ann Hematol. 2016; 95(12):2049-55. doi: https://dx.doi. org/10.1007\%2Fs00277-016-2817-5

15. Vinturache A, Stephenson N, McDonald S, Muci W, Bayrampour H, Tough S. Health - related quality of life in pregnancy and postpartum among women with assisted conception in Canada. Fertil Steril. 2015; 104(1):1-9. doi: http://dx.doi. org/10.1016/j.fertnstert.2015.04.012
16. Hagberg L, Winkvist A, Brekke HK, Bertz F, Johansson EH, Huseinovic E. Cost-effectiveness and quality of life of a dietintervention postpartum: 2-years results from a randomized controlled trial. BMC Public Health. 2019; 19(38):1-10. doi: https://doi.org/10.1186/s12889-018-6356-y

17. Tungchama FP, Piwuna CG, Armiya'u AY, Maigari YT, Davou FJ, Umar MU, et al. Relationship between quality of life and postpartum depression among women in North-Central, Nigeria. Highland Med Res J [Internet]. 2017 [cited Jul 23, 2019]; 17(1):11-8. Available from: https://www.ajol. info/index.php/hmrj/article/view/158114

18. Bodhare TN, Sethi P, Bele SD, Dasari Gayatri MBBS, Vivekanand A. Postnatal quality of life, depressive symptoms and social support among women in Southern India. Women Health. 2015; 55(3):35365. doi: https://doi.org/10.1080/03630242.2014 .996722

19. Souza ABQ, Fernandes BM. Guidelines for nursing care: an effective tool for the promotion of health in puerperium. Rev Rene. 2014; 15(4):594-604. doi: doi.org/10.15253/2175-6783.2014000400006 